\title{
Sur quelques ferrobactéries isolées dans le Sud-Ouest de la France : écologie et rôle dans l'environnement.
}

\author{
J.L. Gouy 1 \\ L. Labroue ${ }^{2}$
}

Mots clés: Ferrobactérics, dénitrification, écologie, environnement.

Les bactéries du fer isolées des eaux et des sédiments du Sud-Ouest de la France sont soit autotrophes (g. Gallionella). soit hétérotrophes (g. Sphaerotilus, Crenothrix, Siderocapsa, etc...).

Les premières sont inféodées à la présence de fer ferreux, alors que les secondes occupent des niches écologiques plus variées, quoique toujours aux limites de stabilité du fer ferreux dans les eaux. $C e$ dernier point, joint à leur exigence en matière organique, facteur de mobilisation du fer, explique leur róle dans le colmatage biologique des drains. De plus, il est probable que l’influence des ferrobactéries déborde largement le cadre du cycle du fer.

Several iron-bacteria isolated in the South-West of France : ecology and role in the environment.

Keywords : Iron-bacteria, denitrification, ccology, environment.

Iron-bacteria isolated from water and sediments of the South.Wcst of France are either autotrophs $(\mathrm{g}$. Gallionella) or heterotrophs (g. Sphaerotilus, Crenothrix, Siderocapsa, etc.).

The first group is linked to the presence of ferrous iron whereas the second group occupies a wide range of ecological niches, though always at the limits of stability for the ferrous iron in the water. This latter point, coupled with their need for organic matter, mobilisation factor of iron. explains their role in the biological clogging of drains. Furthermore, it is probable that the influence of iron-bacteria largely overlaps the limits of the iron cycle.

\section{Introduction}

Les ferrobactéries sont des microorganismes encore mal connus de nos jours ; leur groupe a longtemps été défini comme celui a des bactéries gram négat if qui paraissent utiliser le fer et/ou le manganèse comme un élément essentiel de leur métabolisme énergétique ". En fait, un seul genre du groupe, le genre Gallionella, répond d'une manière stricte à cette définition; les autres genres n'ont, en l'état actuel des connaissances, qu'une relation indirecte avec le fer. Cette diversité au sein des ferro-

1. Laboratoire d'Ecologie Végétale. U.P.S. 39. Atlèes Jules Guesde, 31062 Toulouse Cedex France.

2. Centre d'Ecologie des Ressources Renouvelables, 29, Rue Jeanne Marvig. 31055 Toulouse Cedex France. bactéries se traduit dans leur classification ; ainsi, dans l'édition de 1974 du "Bergey's manual ", les ferrobactéries sont réparties au sein de trois groupes:

- les bactéries engainées

- les bactéries bourgeonnantes et/ou pédonculées

- les bactéries chimiolithotrophes gram négatif.

Nous avons isolé, pendant notre étude, plusieurs genres de ferrobactéries tels Sphaerotilus, Crenothrix, Clonothrix, Siderococcus, Siderosphaera, Naumaniella et Gallionella, présents au niveau régional. Nous avons essayé ensuite, soit par leur culture, soit par l'étude des conditions naturelles dans lesquelles nous les avions observés, de déterminer leurs relations avec le fer et leur rôle dans l'environnement. 


\section{1. - Les principales bactéries isolées}

Elles ont été obtenues à partir d' " ochre » déposé à la sortie ou à l'intérieur de drains, dans des sour. ces, des fossés, des ruisseaux ou des bassins. Les plus « spectaculaires " sont les bactėries engainées.

\section{1. - Les bactéries engainées}

La clé de détermination est la suivante:

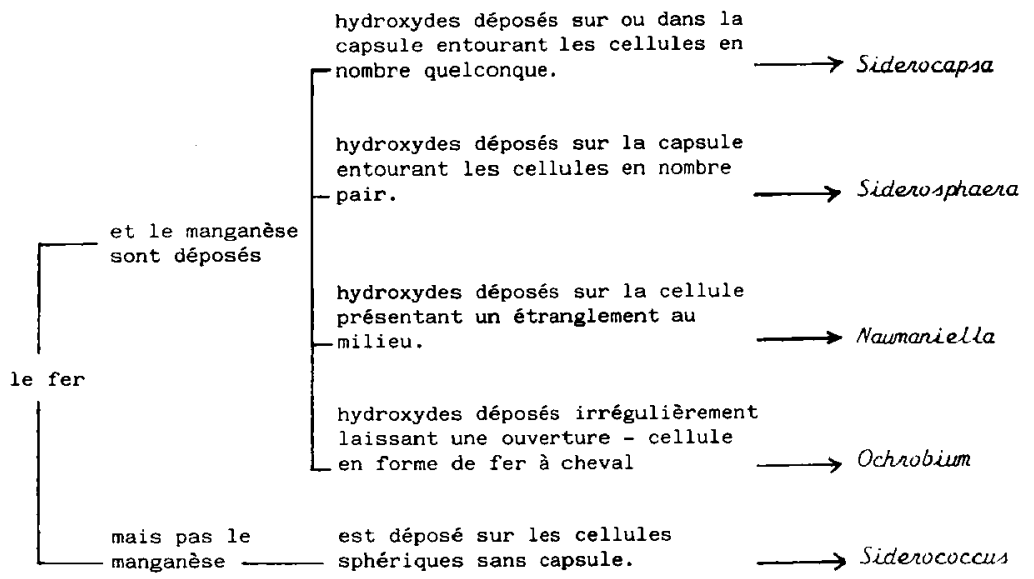

\subsection{1. - Le genre Sphaerotilus}

Le genre Sphaerotilus fut découvert par Kützing, en 1833 dans des rivières polluées ; cette bactérie est en effet caractéristique des zones de poly et mésasaprobie. Elle se présente en général (fig. 1.1) sous forme de filaments (gaines) à l'intérieur desquels se trouvent des cellules en file. Les cellules mesurent de 0,9 à $2,4 \mu$ sur 3 à $10 \mu$; celles-ci sont flagellées en dehors des gaines et la touffe de flagelles (12 à 15) est insérée sur une protubérance subpolaire.

Le genre ne comporte qu'une espèce : Sphaeroti. lus natans.

La bactérie vit fixée, la fixation résultant de la différenciation de la cellule terminale en forme de crampon. Généralement, les gaines paraissent ramifiées en milieu naturel ; en effet, les cellules peuvent sortir, se fixer à l'extérieur de la gaine et donner naissance à une nouvelle gaine, d'où une pseudoramification. La gaine est en général hyaline et de faible épaisseur $(0,07 \mu)$; sa formation dépend de facteurs externes comme :

- la teneur en calcium du milieu: 0,1 mM en $\mathrm{Ca}^{++}$est requise pour sa formation.

- la concentration en éléments carbonés : si le milieu contient plus de $0,5 \%$ de glucose, par exemple, les cellules ne synthétisent plus de gaine; il semble donc que la gaine soit une adaptation aux milieux pauvres en matière organique.

La bactérie que nous avons isolée d'un drain agricole (commune de Lescar, 64), a été cultivée sur le milieu proposé par Stokes (1954). Nous avons noté que certains éléments minéraux jouent un rôle important dans la croissance de la bactérie : outre le calcium, impliqué dans la formation de la gaine, on remarque l'action du phosphate (une concentra- 
tion supérieure à $0,04 \mathrm{M} / \mathrm{l}$ supprime la respiration endogène), et de l'ion ferreux qui inhibe la bactérie aux concentrations assez élevées ( > à $15 \mathrm{mg} / \mathrm{l}$ ) et provoque des déformations cellulaires. A la différence du genre Leptothrix, les dépôts de fer sur les gaines ne sont pas uniformes; on note, de méme, que Sphaerotilus, contrairement à Leptothrix, ne catalyse pas l'oxydation du manganèse.

La bactérie peut tolérer d'assez fortes teneurs en composés organiques et répond positivement à une augmentation de leur concentration.

En ce qui concerne les sources d'azote, Sphaerotilus natans croît en présence d'azote organique, peptone, ou acides aminés.

La bactérie peut également utiliser l'azote minéral $\mathrm{NH}_{4}{ }^{+}, \mathrm{NO}_{3}{ }^{-}$, mais la croissance est moins rapide qu'en présence de peptone.

Les principaux paramètres physico-chimiques qui interviennent sur sa croissance sont :

- la température : elle croît de 5 à $35^{\circ} \mathrm{C}$, son optimum étant compris entre 25 et $30^{\circ} \mathrm{C}$.

- le $\mathrm{pH}$ : entre 5,5 et 8 , la croissance est bonne et l'optimum varie suivant les souches ent re 6,8 et 7,5 .

- l'oxygène : elle est aérobie stricte. Le nitrate ne peut remplacer l'oxygène. Cependant, si en culture pure une baisse de la concentration en $\mathbf{O}_{2}$ nuit à la croissance de la bactérie, en culture mixte, sa résistance l'avantage par rapport à la plupart des autres microorganismes aérobies : c'est ce qui explique la dominance quelquefois très marquée de $S$. $n$. dans les boues activèes. La teneur en oxygène intervient également, comme la teneur élevée en carbone, sur la formation de réserves par la bactérie ; en effet, dans un milieu riche en carbone et (ou) pauvre en oxygène, la cellule accumule de l'acétyl COA, conduisant à la formation du Poly-hydroxy-butyrate (P.H.B.).

\subsection{2. - Le genre Crenothrix (Cohn, 1870)}

Le genre ne comporte qu'une espèce : Crenothrix polyspora isolée pour la première fois en Allemagne.

Les filaments sont fixés au substrat et sont plus larges vers l'extrémité libre. Les pseudoramifications sont rares dans le genre. La gaine est généralement fine, et peut être incrustée par des hydroxydes ferriques à la base.
A l'intérieur de la gaine, les cellules sont de plusieurs sortes : discoidales, cylindriques ou rectangulaires à la base (fig. 1.2).

Vers l'extrémité (fig. 1.2), les cellules se divisent transversalement et longitudinalement et donnent naissance à des conidies, souvent en paquet. La gaine varie en largeur depuis la base $(1,5$ à $4 \mu)$ jusqu'au sommet ( 3 à $9 \mu$ ). Les cellules, suivant les types, varient de 1,3 à $3 \mu$ sur 1,1 à $2,3 \mu$.

La propagation de l'espèce s'effectue par rupture de l'extrémité libre de la gaine et libération des conidies. Dans certains cas, elle peut s'effectuer aussi par libération d'une cellule plus grosse et unique. en bout de gaine.

Le genre est gram négatif, aérobie et organotrophe.

\subsection{3. - Le genre Clonothrix (Roze, 1896).}

Le genre compte actuellement deux espèces : Clonothrix fusca et Clonothrix gracillima. La bactérie se présente sous forme de filaments effilés au sommet, présentant des pseudo-ramifications (fig. 1.3). Les cellules et la gaine sont incolores ou légèrement bleuâtres. La propagation s'effectue de la même manière que chez Sphaerotilus, par rupture de la gaine et libération des cellules.

L'espèce que nous avons isolée présente les caractéristiques suivantes :

Les cellules mesurent de 2 à $2,5 \mu$ sur 12 à $18 \mu$ La gaine est plus large à la base (environ $7 \mu$ ) et effilée au sommet $(<3 \mu)$. Nous avons noté l'existence en bout de filament "d'ampoules " comme l'avait décrit Roze (1896); il semble que ces ampoules se forment par bourgeonnement et, qu'à l'intérieur, il existe des éléments servant à la propagation (Roze 1896). La bactérie vit fixée et nous l'avons isolée sur une algue.

En présence de sels de fer, la gaine bactérienne peut prendre une couleur brune dorée.

La bactérie n'a jamais pu être obtenue en culture pure.

\section{2. - Les Sidérocapsacées}

Les différents genres de la famille n'ont en commun que d'être gram négatif et de déposer des hydroxydes de fer ou de manganèse. La clé de détermination est la suivante: 


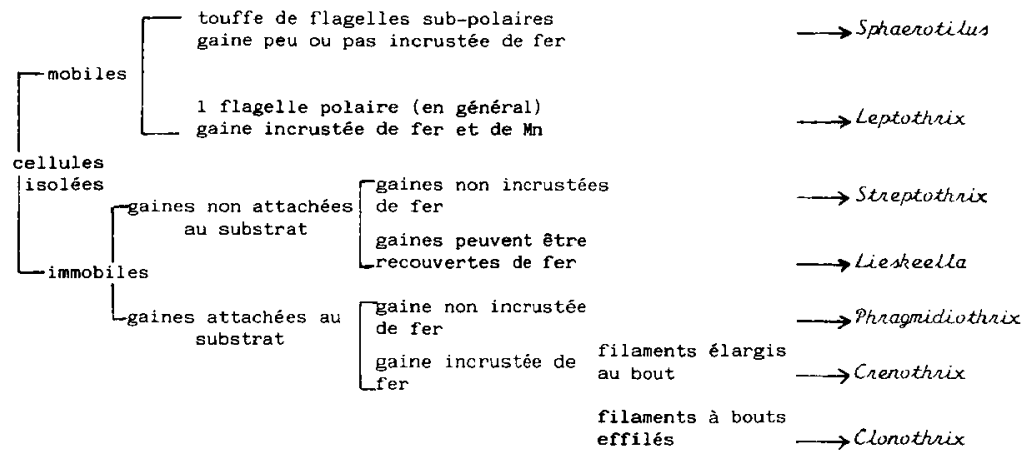

\subsection{1. - Le genre Siderococcus (Dorff, 1934)}

Le genre comprend actuellement deux espèces, Siderococcus limoniticus et Siderococcus communis (fig. 1.4).

Les bactéries se présentent sous forme de petites cellules sphériques de 0,2 à $1,0 \mu$, regroupées en colonies. Les premiers auteurs ont décrit ces cellules comme mobiles mais l'on ne connaît pas le type de flagellation. La multiplication se fait par bour. geonnement, les celíules apparaissant alors comme des poires. Le genre n'a pas de capsule et l'hydroxyde ferrique imprègne toute la colonie plutôt que les cellules isolées. La bactérie n'a pu être cultivée.

\subsection{2. - Le genre Siderosphaera (Beger, 1944)}

Ce genre ne compte qu'une espèce : Siderosphaera conglomerata (fig. 1.5). Il se présente sous forme de petites cellules cocoïdes en paires, entourées d'une capsule primaire. Après division, les nouvelles paires sont entourées par une capsule secondaire et ce. jusqu'à huit paires. La capsule secondaire peut être imprégnée par de l'hydroxyde ferrique. Les cellules mesurent de 1 à $1,2 \mu$; la capsule secondaire peut atteindre $10 \mu$ de diamètre. Les colonies peuvent être de grandes dimensions $(500 \mu)$. La bactérie n'a jamais été cultivée.

\subsection{3. - Le genre Naumaniella (Dorff, 1934)}

Le genre comporte six espèces qui se distinguent par leur aspect morphologique : Naumaniella minor, Naumaniella catenata, Naumaniella elliptica, Naumaniella pygmea, Naumaniella polymorpha, Naumaniella neustonica.

Les cellules sont ellipsoïdales ou bacillaires, à extrémités plus ou moins arrondies, isolées ou en courtes chaines (fig. 1.6). La capsule est fine, sauf aux extrémités, ce qui donne à la cellule l'apparence d'un maillon de chaîne. Les dépôts d'hydroxyde ferrique et quelquefois de manganèse peuvent provoquer l'opacité de la cellule.

Dans un milieu au cit rate ferrique, une culture contenant Naumaniella neustonica, donne un film jaune doré, irisé en surface, et un volumineux dépôt d'hydroxyde ferrique. Toutefois, la bactérie n'a pu être cultivée en culture pure, de sorte que nous ne connaissons pas ses exigences.

Certains auteurs ont signalé qu'une espèce, $\mathrm{Nau}$ maniella polymorpha, était capable d'oxyder le manganèse comme le genre Leptothrix.

\section{3. - Les bactéries pédonculées : le genre Gallionella}

La bactérie fut décrite la première fois par Ehren. berg, en 1836, dans des dépôts fossiles; mais ce furent Cholodny (1924) et Perfil'ev (1926) qui don- 

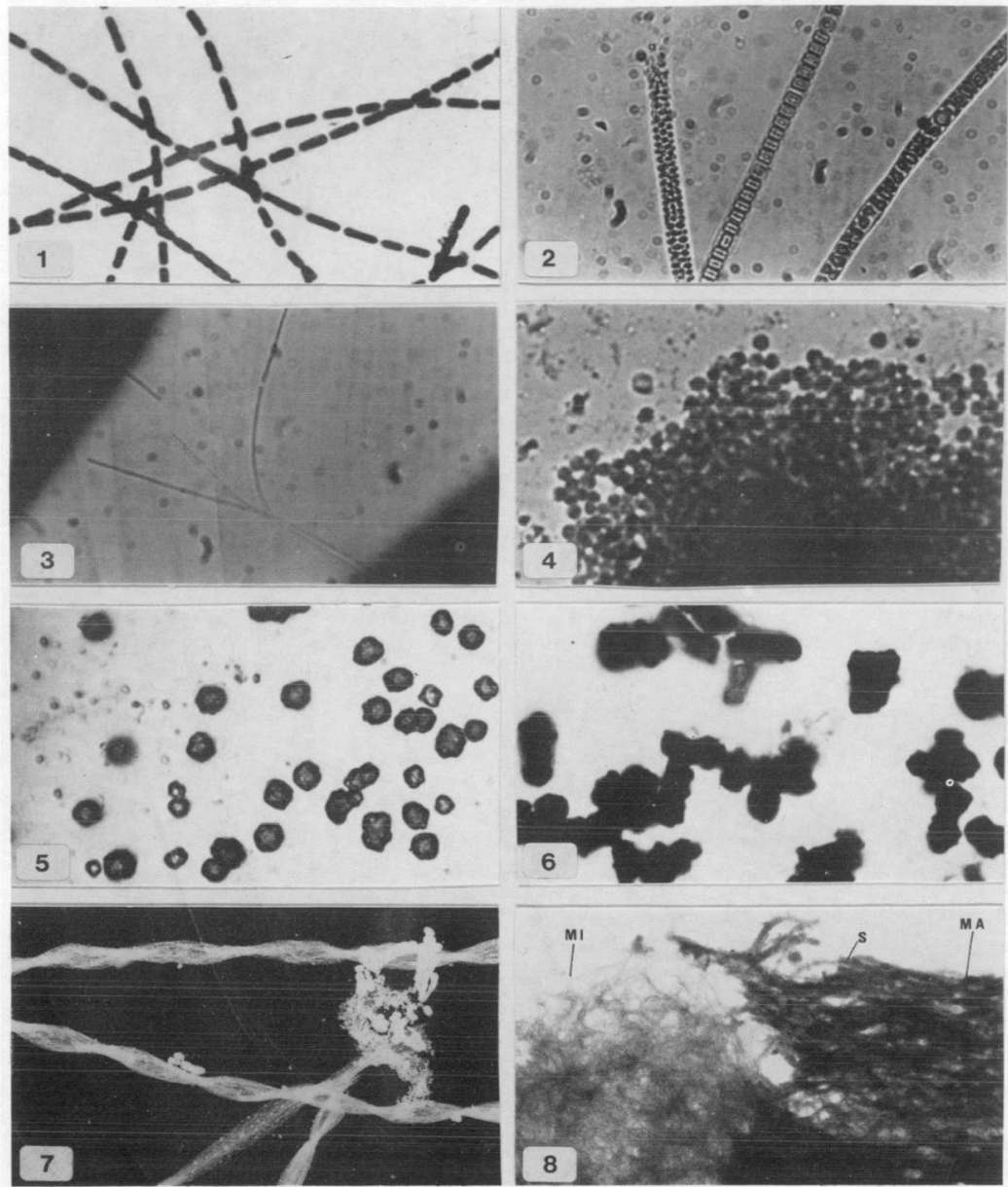

Fig. 1. I: Sphaerotilus natans, forme typique : cellules dans une gaine $\times 1625.2$ : Crenothrix polyspora: les différents types de cellules. $\times 1600.3$ : Clomothrix fusca : pseudo-ramifications de la gaine. $\times 300,4$ : Siderococcus communis. $\times 3800.5$ : Siderosphaera conglomerata. $\times 2400.6:$ Nat maniella netustonica. $\times 2400.7$ : Gallionella ferruginea : aspect torsadé typique de la bande. $\times 5200.8$ : Gallionella ferruginea : structure de la bande: MA macrofibrilles, MI microfibrilles, S segmentation. $\times 68000$. 
nèrent une description complète de sa morphologie et de son cycle de développement. La bactérie était représentée alors comme une cellule en forme de rein dont la partie concave donnait naissance à un pédoncule d'hydroxyde ferrique fixant la bactérie au substrat. Ce fut Van Iterson (1958) qui suggéra, la première, que le "pédoncule " contenait des éléments vivants et était l'élément principal de la bactérie. Ce pédoncule est en fait une bande torsadée constituée de nombreuses fibrilles recouvertes d'hydroxyde ferrique. Cette bande est très caractéristique et permet de diagnostiquer la présence de la bactérie dans son milieu naturel.

A l'heure actuelle, le genre Gallionella compte deux espèces qui se différencient par la taille de la cellule terminale; le nombre et le diamètre des fibrilles constituant la bande :

- Gallionella ferruginea : fibrilles très fines, nombre supérieur à 40 . Cellule terminale 1,2 à $1,5 \mu$ sur 0,5 à $0,7 \mu$.

- Gallionella filamenta: fibrilles plus épaisses, nombre inférieur à 12. Cellule terminale 0,7 à $1,25 \mu$ sur 0,4 à $0,65 \mu$.

La bactérie que nous avons isolée (fig. 1.7.8) correspond à Gallionella ferruginea.

La bande bactérienne présente deux sortes de fibrilles : des microfibrilles de 2,5 à $4 \mathrm{~m}_{\mu}$ et des microfibrilles de 30 à $45 \mathrm{~m}_{\mu}$. Ces fibrilles sont vraisemblablement des stades de développement différents. Au cours de not re culture de la bactérie, nous n'avons pas mis en évidence de cellule terminale en forme de rein ; cependant, nous avons noté la présence constante de nombreux « bourgeons » fixés le long de la bande. Ces bourgeons se présentaient soit isolés, soit en amas, et leur taille pouvait atteindre $0,5 \mu$ de diamètre. Il semble qu'ils jouent un rôle dans la propagation de l'espèce.

Au niveau structural, la bactérie présente plusieurs particularités : outre l'absence de peptidogly. cane (Balashova 1969), la bactérie possède une relation très étroite avec le fer. Celui-ci peut être mis en évidence par la coloration de Meyer mais cette coloration est moins nette qu'avec des dépôts " purs " d'hydroxyde ferrique, ce qui laisse supposer que le fer est engagé dans une liaison forte avec les fibrilles de la bactérie.

La culture de la bactérie s'effectue dans le milieu proposé par Kucera \& Wolf (1957). La source d'énergie étant un précipité de FeS, le milieu est sélectif car il ne contient pas d'élément carboné. L'azote est apporté sous forme ammoniacale. La bactérie est autotrophe stricte comme l'a montré Hanert en 1968 : elle incorpore le $\mathrm{C}^{14}$ en présence de $\mathrm{C}^{14} \mathrm{O}_{2}$ dans le milieu.

La bactérie catalyse la réaction suivante: $\mathrm{Fe}^{2+} \rightarrow \mathrm{Fe}^{3+}+\mathrm{e}^{-} \quad \Delta \mathrm{G}^{\prime} \mathrm{o}=-11 \mathrm{kcal}$. et récupère une partie de cette énergie pour réduire et incorporer le $\mathrm{CO}_{2}$.

Les autres paramètres importants du milieu de culture sont les suivants :

- L'oxygène : compte tenu de l'oxydabilité du fer ferreux en présence d'O $\mathrm{O}_{2}$, celui-ci est un facteur limitant au développement de la bactérie. L'oxydation chimique du fer étant beaucoup plus rapide que l'oxydation biologique, la bactérie est microaérophile.

- Le pH : il ne semble pas jouer de rôle direct sur la bactérie. En effet, celle-ci a été isolée d'eaux légèrement acides $(\mathrm{pH} \quad 6,0)$ et légèrement alcalines $(\mathrm{pH}$

7,5). Toutefois, le $\mathrm{pH}$ intervient d'une manière indirecte, en tant qu'élément régulateur de la solubilité du fer.

- La température : la bactérie est psychrophile ; sa croissance s'arrête quand la température dépasse $25^{\circ} \mathrm{C}$. Son développement semble optimal entre 5 et $15^{\circ} \mathrm{C}$.

\section{2. - Caractères généraux des ferro- bactéries}

Il ressort de cette étude que le groupe des ferrobactéries est extrêmement varié et que le seul point commun à tous les genres soit la présence d'hydroxyde ferrique sur les cellules.

Pour que cette étude soit complète, il est nécessaire de tenir compte de deux genres que nous n'avons pu mettre en évidence, le genre Thiobacillus et le genre Leptothrix. Le premier est autotrophe et l'espèce ferroxidans tire son énergie de l'oxydation du fer ferreux; son écologie est celle des eaux très acides. Le second genre est hétérotrophe, comme l'ont mont ré de nombreuses études. On a mis en évidence sa capacité à oxyder le manganèse, mais il ne semble pas que la bactérie récupère l'énergie de cette oxydation pour son métabolisme (Mudler 1972). 
Donc, si nous passons en revue les membres de ce groupe des ferrobactéries, nous voyons que s'ils diffèrent par leur morphologie, ils diffèrent aussi par leur répartition en fonction des facteurs du milieu.

\section{1. - Répartition en fonction des facteurs du milieu}

- le pH : très acide pour Thiobacillus, peu acide à neutre pour les autres genres

- la température: optimum $15^{\circ} \mathrm{C}$ Gallionella optimum entre 15 et $20^{\circ} \mathrm{C}$ Thiobacillus optimum entre 20 et $25^{\circ} \mathrm{C}$ Leptothrix

optimum $25^{\circ} \mathrm{C}$ Sphaerotilus

- l'oxygène : aérobies strictes: Sphaerotilus Thiobacillus microaérophiles: Gallionella, Leptothrix, Siderococcus ou anaérobies facultatives.

- la teneur en fer ferreux: Thiobacillus croit dans une eau contenant plusieurs grammes de fer par litre.

- Gallionella dans

une eau contenant plusieurs dizaines de $\mathrm{mg} / \mathrm{l}$. Leptothrix, Side. rococcus, Naumaniella: plusieurs mg. Sphaerotilus, Clo-

nothrix, Crenothrix se trouvent dans les eaux pouvant ne pas contenir de fer ferreux.

\section{2. - Relations avec le fer}

En ce qui concerne la relation des ferrobactéries avec le fer, il faut distinguer plusieurs cas. Tout d'abord, celles qui sont autotrophes: Gallionella et Thiobacillus (ferroxidans) et qui utilisent le fer comme source d'énergie. Dans ce cas, la relation est stricte et la bactérie ne croît pas sans fer ferreux et ces genres représentent des ferrobactéries « sensu stricto".

A l'opposé, il existe un certain nombre de genres, Leptothrix, Sphaerotilus, Clonothrix, Crenothrix, qui sont hétérotrophes et n'utilisent pas le fer ferreux comme source d'énergie. Cependant, pour certains genres, en particulier Leptothrix, il faut souligner que les eaux dans lesquelles on les rencontre sont pauvres en matière organique et la teneur en métal réduit n'est pas négligeable. Il y aurait, au moins dans ce cas, une adaptation de la bactérie à cet environnement particulier.

Enfin il existe un groupe de bactéries, les Sidérocapsacées, encore mal connues car non cultivées, que l'on rencontre dans des conditions limites de stabilité du fer ferreux (faible pression d' $\mathrm{O}_{2}$ en particulier) et, dans ce cas, il serait possible que quelques genres puissent tirer une partie de leur énergie de l'oxydation du fer ferreux.

\section{3. - Les milieux de prédilection}

Le mieux connu est celui de Sphaerotilus natans, puisque cette bactérie est un indicateur de pollution organique. Il faut noter, toutefois, que la fixation et la présence de la gaine permettent à la bactérie de vivre dans des eaux courantes, peu riches en matiere organique; mais c'est surtout dans les cours d'eau où il y a des rejets organiques (laiteries, raffineries, papèteries, brasseries, égouts) que l'on rencontre le plus fréquemment la bactérie. En aval de tels rejets, Sphaerotilus natans peut avoir une croissance explosive et devenir dominante. Les études de Curtiss et Curds (197 1) in Dondero (1975), montrent qu'elle est souvent associée à d'autres organismes comme Beggiatoa, Leptomitus, Fusarium, Flavobacteritm, Chromatium, Spirillum, Lamprocytis, Azotobacter, Chlorella, Euglena, etc...

Le genre Crenothrix a une répartition large, dans les eaux courantes, mais aussi stagnantes contenant de la matière organique et, quelquefois, des sels de fer.

La bactérie est souvent fixée, cornme le genre Clonothrix, sur des algues vertes. On remarque, de même, qu'il n'y a que quelques genres d'algues comme les Vatcheria quj portent ces bactéries.

Le fort développement de la bactérie conduit à des masses brunâtres d'assez grande importance. Différents auteurs, tels Barbic et Bracilovic (1974), signalent la présence de cette bactérie dans des drains et dans des puits où, par son développement, elle réduit la circulation d'eau.

Chez les Sidérocapsacées, le genre Siderococcus est largement représenté dans les eaux douces ; on le trouve généralement dans les sédiments, mais aussi dans les boues de fossés où nous l'avons isolé. Il est fréquent dans les eaux neutreset peut supporter des eaux alcalines (nous l'avons isolé de boues 
traitées à la chaux dont le pH était de 8 environ). L'étude de Dubinina et al. (1971), dans les étangs et les lacs d'URSS, montre que le genre est réparti dans des zones où la concentration en oxygène est faible $(0,5$ à $1 \mathrm{mg} / \mathrm{l})$, où le fer ferreux est présent, mais également ou le fer ferrique devient important, ce qui indique une zone limite au-delà de laquelle le fer ne se trouve plus qu'à l'état ferrique.

Le genre Side rosphaera est présent dans les sédiments des fossés, des étangs et des cours d'eau.

Le genre Naumaniella peut se rencontrer soit à la surface des eaux, soit dans les sédiments des cours d'eau contenant du fer ferreux, généralement en association avec Gallionella et Leptothrix.

Nous avons mis en évidence le genre Gallionella à la sortie d'un drain dans la commune de Capvern (Hautes-Pyrénées - 65).

L'eau naturelle présentait les caractéristiques suivantes :

Température : $1114,5^{\circ} \mathrm{C} ; \mathrm{pH}: 5,90-6,30$; Eh : $+64-+90 \mathrm{mv}$; Conductivité : $132 \mu \mathrm{m}_{\text {hos }} \mathrm{cm}^{-1}$; Débit : 3,5-5 l/min. : $\mathrm{O}_{2}: 0,47 \mathrm{mg} / \mathrm{l} ; \mathrm{Fe}^{2+}$ : $19,60 \mathrm{mg} / \mathrm{l} ; \mathrm{Mn}: 0.46 \mathrm{mg} / \mathrm{l} ; \mathrm{Ca}^{++}: 27 \mathrm{mg} / \mathrm{l} ; \mathrm{K}^{+}$: $0,36 \mathrm{mg} / \mathrm{l} ; \mathrm{Na}^{+}: 4,90 \mathrm{mg} / 1 ; \mathrm{Mg}^{++}: 1,75 \mathrm{mg} / \mathrm{l}$; $\mathrm{SO}_{4}^{-}: 18,8 \mathrm{mg} / \mathrm{l}$; $\mathrm{HCO}_{3}^{-}: 107.6 \mathrm{mg} / \mathrm{l} ; \mathrm{Cl}^{-}$: $15,24 \mathrm{mg} / 1$; $\mathrm{NH}_{4}^{+}: 2,40 \mathrm{mg} / \mathrm{l}$; $\mathrm{NO}_{3}^{-}: 0,24 \mathrm{mg} / \mathrm{l}$; $\mathrm{PO}_{4}^{3-}$ : $0,005 \mathrm{mg} / \mathrm{l}$.

L'eau ne présentait ni odeur ni couleur et pourtant l'on remarque l'importance des teneurs en fer alors que le carbonne ne dépasse pas $2.4 \mathrm{mg} /$. Cependant, ce carbone correspond essentiellement à des acides aminés, en particulier de la cystathionine qui représente, en nmoles, 74 p. 100 de ces derniers. L'isolement de ces bactéries à partir des drains dont elles provoquent le colmatage biologique amène à stinterroger sur leur rôle dans l'environnement.

\section{3. - Le rôle des ferrobactéries dans l'environnement}

Le colmatage ferrique des puits, des canalisations et des drains est un phénomène connu depuis l'antiquité, mais c'est seulement depuis 150 ans, environ, que le rôle prépondérant de certaines bactéries dans le processus fut décrit. Les espèces les plus citées dans les cas de colmatage ferrique sont : Leptothrix, Gallionella, Sphaerotilus, Siderocapsa et, dans une moindre mesure, Toxothrix, Crenothrix, Clonothrix, Siderococcus, Naumaniella.

Ces bactéries (Sidérocapsacées, Gallionella, Thiobacillus) pourraient être impliquées dans l'initiation de "nodules » ferriques et donc dans la génèse des gisements de fer.

En fait, les expériences permirent de distinguer deux groupes de bactéries : celles qui oxydent le fer ferreux (quelques espèces) et celles qui le précipitent en dégradant le ligand organique (un grand nombre de bactéries des sols et des eaux). Les premières, telles Gallionella ferruginea, ont, à priori, une action spécifique. Elles vivent dans des milieux aquatiques dans lesquels le fer ferreux est relativement stable en solution ( $\mathrm{pH}$ acides, Eh faibles, $\left[\mathrm{O}_{2}\right]$ voisine de 0 ). La présence de la bactérie dans de tels milieux peut provoquer d'importants dépôts d'hydroxyde ferrique, alors que les conditions physico-chimiques du milieu ne le permettraient pas. Ces bactéries jouent donc un rôle important dans l'environnement et dans l'aménagement (colmatage ferrique de drains).

Gallionella possède, par ailleurs, une caractéristique importante qui est de pouvoir, dans certaines conditions, utiliser l'ion nitrate à la place de l'oxygène comme accepteur terminal d'électron. Compte tenu du milieu dans lequel elle vit (présence d'ion ferreux), le nitrite est rapidement réduit conduisant à de l'azote gazeux (Gouy et al. 1983).

Ce phénomène de dénitrification est très intéressant car il est le fait d'une espèce au totrophe, ce qui est relativement rare dans la nature. Cette dénitrification autotrophe cont ribue peut-être à l'épuration des horizons profonds des sols et des nappes souterraines pauvres en matière organique.

Les bactéries hétérotrophes (les plus nombreuses) peuvent croitre dans des milieux ne contenant que des traces de fer ferreux et leur rôle déborde largement le cadre du cycle du fer : c'est le cas de Sphaerotilus natans. L'infestation par Sphaerotilus natans entraine de nombreux dommages au niveau de la faune benthique (altération de son habitat par la boue formée) avec une action sur les poissons qui consomment cette faune et une action sur les cufs (pourrissement). Sphaerotilus natans peut intervenir au niveau de la nitrification, ainsi que l'a noté Reinheimer (1959, in Dondero, 1975) : la forte croissance de la bactérie entraîne une déficience en $\mathrm{PO}_{4}^{-}$, ce qui a pour effet de retarder la nitrification et donc d'augmenter la teneur en $\mathrm{NH}_{4}{ }^{+}$de l'eau. 
Toutefois, il existe aussi, dans la littérature, des exemples d'action bénéfique de la croissance de Sphaerotilus natans. Warren C.E. (1971 in Dondero 1975) rapporte que l'enrichissement en sucre d'un tronçon de rivière entraîna une production importante de Sphaerotilis natans qui servit de nourriture à diverses larves d'insectes (chironomides. entr'autres). Par la suite, on observa une production de truites plusieurs fois plus importante que celle d'un tronçon de rivière non enrichi en sucre.

Outre son rôle dans les rivières, la bactérie se trouve impliquée dans d'au tres phénomènes comme le " bulking " dans les stations d'épuration. Ce processus apparaît pendant certaines phases du traitement des eaux usées et le mécanisme fut décrit par Adamse, en 1968: "Au cours du trajtement des M.O., la décomposition des composés organiques entraîne une baisse du $\mathrm{pH}$ et une chute de la teneur en $\mathrm{O}_{2}$ (apparition d'acides intermédiaires). Quand ces acides sont consommés, le pH remonte, l'oxygène dissous réapparaît et la décomposition des protéines commence à relâcher des composés alcalins. Au cours de la première phase, Sphaerotilus natans supplante les autres bactéries car elle utilise mieux l'azote organique et peut se contenter d'une faible tension en oxygène. Si ces conditions sont maintenues, le processus de non sédimentation des boues (bulking) commence ".

Enfin, la bactérie se trouve impliquée dans divers phénomènes de colmatage des puits (colmatage des grilles), des conduites d'eau non traitée, et surtout des drains agricoles dans certaines régions (Cullimore \& Mc Cann, 1977, Ford 1979, Ivarson \& Sojak 1978). L'infestation a lieu principalement dans le cas de drains posés dans des sols organiques et lorsqu'il $\mathrm{y}$ a des apports exogènes de matière organique vers les drains (élevage intensif, pâture du bétail dans les champs après récolte...). Le colmatage de drain agricole que nous avons étudié confirme ces conditions en ce sens que le drain était posé dans une zone d'an. ciens marais et que les eaux arrivant au drain étaient chargées en matière organique. Dans ce cas (commune de Lescar - 64), le colmatage fut très rapide : environ un mois après sa pose, le drain ne coulait plus.

\section{Conclusion}

Toutes les bactéries oxydant ou précipitant le fer, de même que celles qui le réduisent, vont intervenir dans l'exclusion temporaire $d u$ fer dans les cycles biologiques de transformation, et, peut-être, dans la formation des gisements.

Certaines espèces comme Thiobacillus ferroxidans et Gallionella ferriginea vont oxyder le fer, le rendant ainsi insoluble. Ces deux espèces ne sont pas rencontrées ensemble car les conditions favorables à leur développement diffèrent sensiblement.

En association, quelquefois, avec ces bactéries autotrophes, de nombreux microorganismes hétérotrophes du sol et des eaux vont participer à la précipitation du fer en détruisant la partie organique du complexe fer - matière organique. C'est le cas des genres Sphaerotilus, Leptothrix, Siderocapsa... qui participent au colmatage des drains. De nombreux auteurs ont montré que l'habitat de ces bactéries correspondait à des zones où la teneur en oxygène pouvait être voisine de zéro, ce qui explique leur présence à l'inter-face sol drain. Le fer libéré de son vecteur organique est oxydé chimiquement. Connaissant le $\mathrm{pH}, \mathrm{l}$ 'Eh et les teneurs en fer et en matière organique de l'eau, il est possible, dans une certaine mesure, d'estimer le risque de colmatage ferrique sur différents sites.

Enfin nous avons montré l'intervention de Gallionella ferruginea dans le cycle de l'azote. Cette bactérie et d'autres pourraient intervenir dans la purification des eaux vis-à-vis du phosphore en l'insolubilisant sous forme de complexe fer ferrique phosphate.

Ce groupe des ferrobactéries est intéressant à maints points de vue et il est important d'étudier les genres qui, jusqu'ici, n'ont fait l'objet que de descriptions morphologiques. 


\section{Travaux cités}

Adamse (A.D.). 1968. - Response of dairy waste activated sludge to experimental conditions affecting $\mathrm{pH}$ and dissolved oxygen concentration. Water Research, $2: 703-713$.

Balashova (V.V.) 1969 . - Taxonomy of the genus Gallionella Microbiology, 37 : 590.598, Translated from Russian.

Barbic (F.F.) \& Bracilovic (D.M.). 1974 - Iron and manganese bacteria in ranney wells. Water Research. I : 895-898.

Cullimore (D.R.) \& Mc Cann (A.). 1977. - The idertification, culti. vation and control of irom bacteria in ground water. In "Aquatic Microbiology n. F.A. Skinner \& J.M. Shewan (Ed.) Acade. mic Press, London : 219-261.

Curtis (E.J.C.j \& Curds (C.R.). 1971. - Sewage fungus in rivers in the united kingdom : the slime commanity and its constituent organisms. Woter Research, $5: 1147.1159$.

Dondero (N.C.). 1975, - The Sphaerotilus-Leptothrix group. Anmual Review of Microbiology, $29: 407-428$.

Dubinina (G.A.) \& Deryugina (Z.P.). 1971. - Electron microscope study of iron manganese concretions from lake Punnus-Yarvi. Doklavi Biological Scierces, 201 : 738-740.
Ford (H.W.). 1979. - Caracteristics of slime and ochre in drainage and irrigation systems. Transactions of the ASAE 2, 16: 1093-1096.

Gotoh (S.) \& Patrick (W.H.). 1972. - Transformation of manganese in a water-logged soil as affected by redox potential and $\mathrm{pH}$. Soil Sci. Soc. Amer. Pr., 36: 738.741 .

Gouy $($ J.L.) , Berge (Ph.) \& Labroue (L.). 1983. - Gallionella ferruginea facteur de dénitrification dans les eaux pauvres en matiere organique. C.R. Acad. Sc. Paris (sous presse).

Hanert (H.). 1968. - Untersuchungen zur Isolierung, Stoffwechselphysiologie und Morphologie von Gallionella ferruginea Ehrenberg. Archiv: fur Mikrobiologie, 60 : 348-376.

Ivarson (K.C.) \& Sojak (M.) 1978 - Microorganisms and ochre deposits in field drains of Ontario. Can. J. Soil Sci. $58: 1.17$.

Kucera (S.) \& Wolfe (R.S.) 1957. - A selective enrichment method for Gallionella ferruginea. J. Bacteriol, 74 : 344.349

Mulder (E.G.). 1972. - Le cycle biologique tellurique et aquatique du fer el du manganèse. Rev. Ecol. Biol. Sol., IX : 321-348.

Siokes (J.L.). 1954. - Studies on the filamentous sheathed iron bacterium Sphaerotilus natans, J. Bacteriol., $67: 278-291$.

Van Veen (W.L.), Mulder (E.G.) \& Deinema (M.H.). 1978. - The Sphaerotilus-Leptothrix group of bacteria. Microbiological Reviews, 42 : 329-356. 